\title{
RELATIVISTIC CONSIDERATIONS FOR PRECESSION AND NUTATION
}

\author{
S.A. KLIONER AND M. SOFFEL \\ Lohrmann Observatorium, Technische Universität Dresden, \\ Mommsenstraße 13, D-01062 Dresden, Germany
}

\begin{abstract}
The whole scope of problems related with the rotational motion of celestial bodies is briefly discussed. Relativistic modeling of the Earth rotation is considered from a conceptual point of view. Relativistic effects in rotational equations of motion of an extended body in general relativity are discussed. Numerical values of the effects are given.
\end{abstract}

\section{What do we call Earth rotation and how do we model it?}

Definition of rotational motion of an extended body being fairly simple in Newtonian mechanics is not obvious at all in the framework of general relativity. Theoretically speaking the concept of a precessing extended rigid body in general relativity encounters fundamental difficulties and cannot be introduced even in the first post-Newtonian approximation. From a practical point of view, however, the rotational motion of the Earth even at the Newtonian level is defined operationally through the time-dependence of geocentric quasi-inertial coordinates of observing sites. An analogous operational definition can be applied in general relativity. To this end, we need a physically adequate geocentric reference system to model local physics of the Earth. Then time-dependence of coordinates of observing sites relative to that geocentric reference system gives relativistic operational definition of the rotation of the Earth.

\section{Relativistic reference systems}

Nowadays there are two well developed formalisms for the construction of relativistic astronomical reference systems: the Brumberg-Kopeikin formalism (see, e.g., Brumberg, 1991) and the DSX formalism (Damour, Soffel, Xu, 1991, 1992, 1993). Although the two approaches look quite different at first glance, the main serious difference between them is the concept of mass multipole moments of some gravitating body. Here, the DSX formalism makes use of the so-called Blanchet-Damour moments that have direct physical significance for an isolated body. It is mainly because of this fact that the DSX scheme is superior to the Brumberg-Kopeikin formalism.

The two principal reference systems needed to model the Earth rotation are the barycentric reference system of the solar system and the local geocentric reference system.

The barycentric reference system is natural to model translational motion of the center of mass of the Sun and planets as well as light propagation from the distant sources (planets, stars or quasars) to the observers in the solar system. This reference system is used, for example, to construct the JPL planetary ephemerides and to define the International Celestial Reference System (ICRS).

In principle, any reference system covering a region of space-time under consideration can be used to model any kind of physical phenomena in that region. However, in some reference systems the mathematical description of physical processes is simpler than in others. Moreover, relativistic models involve a set of parameters (say, masses of planets, coordinates of observing stations, coordinates of planets or satellites at some initial moment of time) which are usually defined only with respect to that reference system. Nevertheless, it is usually preferable if these parameters could be reasonably interpreted from a physical point of view. A local geocentric reference system is physically adequate to model physical phenomena occurring in the vicinity of the Earth. Influence of the external masses (the Sun, planets, etc.) reduces in this reference systems to tidal effects. This 
makes a mathematical description of local physical phenomena in this reference system especially simple. On the other hand, coordinate parameters defined in that local reference system could then be physically interpreted at some reasonable level of accuracy in terms of local physical phenomena and without the necessity to remember always their relativistic coordinate nature (e.g., time dependence of the geocentric coordinates of observing sites can be interpreted as a result of the rotational motion of the Earth and some geophysical drifts). The local geocentric reference system is used to describe translational motions of Earth's satellites, to model Earth rotation (including precession-nutation angles), and therefore, to define the International Terrestrial Reference System.

Both reference systems are defined by the structure of their metric tensors. Coordinate transformations between the reference systems are indispensable to relate coordinates of objects, bodies and observers defined in those two reference systems and are given in detail in the formalisms.

\section{Precession-nutation angles}

Newtonian theory of Earth rotation heavily uses various angles between various planes and lines, and their time-dependence to describe the Earth's rotation. The concept of a plane, a line and an angle between them can be meaningfully introduced only in Newtonian physics based upon Euclidean space. Curved pseudo-Riemannian manifolds that we are dealing with in general relativity do not allow us to consider planes, lines and angles in a physically meaningful way. However, forgetting about physical meaning we can use three space coordinates of some reference systems to introduce "coordinate planes", "coordinates lines" and "coordinates angles" formally in the same way as we do in Euclidean geometry. Those concepts being defined only in one reference system can still be useful from a formal mathematical point of view.

By its construction the local geocentric reference system is physically adequate for modeling of any physical processes occurring in the vicinity of the Earth, and in particular, for modeling the rotation of the Earth itself. In this geocentric reference system $(T, X, Y, Z)$ for the sake of continuity the coordinate plane $\left(T=T_{0}, Z=0\right)$ may, e.g., be related with some equator at some epoch $T_{0}$. The vectors of the post-Newtonian spin, angular velocity or figure axes (see below) then define the precession-nutation angles in that system. The coordinate transformations between the geocentric and barycentric reference systems enable one to define the orientation of the Earth with respect to distant celestial objects.

On the other hand, the concept of a coordinate plane has fundamental restrictions as compared to its Newtonian (Euclidean) counterpart. Thus, a coordinate plane is defined in one specific reference system. If we transform that plane into some other reference system it would be no longer a coordinate plane in that other reference system. Some agreements could be adopted to work with coordinate planes defined in different reference systems (see, e.g., Brumberg, Bretagnon, Guinot, 1996). However, for example, the concept of time-dependent plane (say, moving ecliptic) cannot be defined with the post-Newtonian accuracy in a reasonable way.

\section{Rotational equations of motion}

In the local geocentric reference system one can derive rotational equations of motion of the Earth, which take the same functional form as in Newtonian physics

$$
\frac{d}{d t} S^{i}=L^{i}+\mathcal{O}\left(c^{-4}\right)
$$

where $S^{i}$ is the post-Newtonian spin, and $L^{i}$ is the post-Newtonian tidal torque (see, e.g., Damour, Soffel, Xu, 1993 and Klioner, 1996 for details). These equations are valid without any assumption on shape and composition of the Earth. Both spin and torque coincide with their Newtonian counterparts in the Newtonian limit. The torque $L^{i}$ vanishes for case of an isolated body. Both $S^{i}$ and $L^{i}$ contain many relativistic contributions. However, in practice we determine the value of $S^{i}$ directly from observations rather than compute it from the distribution of density, pressure, etc. within the Earth. Therefore, only the contributions in the torque are important for practical applications. One can show (see, Damour, Soffel, $\mathrm{Xu}, 1993$ ) that $L^{i}$ can be expanded as 


$$
L^{i}=\sum_{l=0}^{\infty} \frac{1}{l !}\left(\epsilon_{i a b} M_{a L} G_{b L}+\frac{1}{c^{2}} \frac{l+1}{l+2} \epsilon_{i a b} S_{a L} H_{b L}\right),
$$

where $L=i_{1} i_{2} \ldots i_{l}$ is the multiindex, $M_{L}$ are the Blanchet-Damour mass multipole moments, $S_{L}$ are spin moments needed to Newtonian accuracy only, and $G_{L}$ and $H_{L}$ are the electric- and magnetic type tidal moments of external gravitational field. Both $M_{L}$ and $S_{L}$ are parameters of the gravitational field of the Earth and should be determined from observations. The moments $G_{L}$ and $H_{L}$ can be computed from planetary and lunar ephemerides. For practical purposes the expansion (2) can be truncated as usual considering magnitudes of the multipole and tidal moments.

One should note, that the post-Newtonian spin and the rotational equations of motion (1) are not unique (see, Damour, Soffel, Xu, 1993 for a discussion). Therefore, in practice one has to adopt a definition of spin by some agreement. Say, the form of expansion (2) of the torque $L^{i}$ makes the definition of $S^{i}$ unique.

\section{Angular velocity and tensor of inertia}

Eq. (1) is sufficient to discuss precession and nutation of the spin. For many reasons it is also important to consider precession and nutation of the angular velocity and figure axis. Therefore, we need a relativistic definition of the tensor of inertia and angular velocity.

A variety of theoretical approaches can be followed to get the same post-Newtonian definition of the tensor of inertia $C^{i j}$ (from restricted rigid body models (Soffel, 1994) to relativistic Tisserandlike axes of a deformable Earth (Klioner, 1996)). This allows one to split the spin $S^{i}$ into a product of the tensor of inertia and corresponding angular velocity

$$
S^{i}=C^{i j} \omega^{j}
$$

The post-Newtonian tensor of inertia $C^{i j}$ is defined as integral over the volume of the body. Again, although the definition of $C^{i j}$ contains a number of explicit relativistic terms usually we do not compute $C^{i j}$ from the distribution of density, pressure, etc. within the Earth, but determine the values of $C^{i j}$ from observations. Therefore, for practical purposes we can simply use the fact that the spin $S^{i}$ can be represented in the form given in (3).

The definition of angular velocity of a deformable body is not unique already in Newtonian mechanics (one can consider Tisserand axes and principal axes of inertia which give two different definitions of angular velocity). This ambiguity is aggravated in general relativity by the ambiguity in the definition of the relativistic tensor of inertia $C^{i j}$ for the non-isolated Earth. Therefore, one more agreement is needed.

\section{Rigid-multipole model}

A rigid Earth plays a very important role as a first order approximation in the Newtonian theory of Earth's rotation. This rigid model (1) crucially simplifies the mathematical description of the rotational motion and (2) is not too far from reality, so that the effect of non-rigidity can be than added to the model by means of perturbation theory. The reason why the rigid body model substantially simplifies the rotational equations of motion is that both the mass multipole moments $M_{L}^{\text {Newt }}$ and the tensor of inertia $C_{\text {Newt }}^{i j}$ rotate rigidly. In other word there exist a rigidly rotating reference system $Y^{i}=P^{i j}(T) X^{j}$, where $P^{i j}(t)$ is some time-dependent orthogonal matrix, where both $M_{L}^{\text {Newt }}$ and $C_{\text {Newt }}^{i j}$ are constant.

This nice feature can obviously be saved in the post-Newtonian framework. One might define "rigid-multipole" models by means of a series of assumptions

$$
\begin{aligned}
& C^{i j}(t)=P^{i a} P^{j b} \bar{C}^{a b}, \quad \bar{C}^{i j}=\operatorname{diag}(\mathcal{A}, \mathcal{B}, \mathcal{C}), \quad \mathcal{A}, \mathcal{B}, \mathcal{C}=\text { const }, \\
& M_{i_{1} i_{2} \ldots i_{l}}=P^{i_{1} a_{1}} P^{i_{2} a_{2}} \ldots P^{i_{l} a_{l}} \bar{M}_{a_{1} a_{2} \ldots a_{l}}, \quad \bar{M}_{a_{1} a_{2} \ldots a_{l}}=\text { const, } \quad l \geq 2, \\
& S_{i_{1} i_{2} \ldots i_{l}}=C^{k i_{1} i_{2} \ldots i_{l}} \omega^{k}, \quad l \geq 2, \\
& C^{k i_{1} i_{2} \ldots i_{l}}=P^{k j} P^{i_{1} a_{1}} P^{i_{2} a_{2}} \ldots P^{i_{l} a_{l}} \bar{C}^{j a_{1} a_{2} \ldots a_{l}}, \quad \bar{C}_{a_{1} a_{2} \ldots a_{l}}=\text { const, } \quad l \geq 2,
\end{aligned}
$$


and the orthogonal matrix $P^{i j}(t)$ is related to the angular velocity $\omega^{i}$ from (3)

$$
\omega^{i}(t)=\frac{1}{2} \varepsilon_{i j k} P^{m j}(t) \dot{P}^{m k}(t)
$$

Relations (6)-(7) for the higher spin moments $S_{L}, l \geq 2$ and for $C_{i L}$ are only necessary to Newtonian accuracy since they appear only in relativistic terms of (2).

Note, that in Newtonian theory we derive or prove (4)-(8) from the fundamental property of rigidity of the body. Here, on the contrary, in general relativity we define the model by assuming the properties of $C^{i j}, M_{L}$ and $S_{L}$ not considering some more fundamental property of the body.

The experience of the Newtonian models of Earth's rotation shows that the phenomenological model (4)-(8) can be used as a first-order approximation for a description of the global rotational motion of the Earth. As in Newtonian theory, the model is a basis for considering the effects of non-rigidity in the rotation of the Earth as a whole. However, the model does not constrain at all internal structure and dynamics of the Earth and completely insufficient for, e.g., a post-Newtonian theory of elasticity, which still waits for a rigorous treatment.

\section{Observational consequences}

There are three main observational consequences of general relativity in the rotation of the Earth:

1. In practice we prefer to use so-called kinematically nonrotating geocentric reference system which rotates relative to a locally inertial reference system. This results in Coriolis and centrifugal forces in the right-hand side of (1). These forces gives additional effects in the orientation of the spin of the Earth which are called geodetic precession $\left(\sim 1.9^{\prime \prime}\right.$ per century) and nutation (with an amplitude of 0.15 mas and a period of one year). (see, e.g., Bizouard, et al., 1992 for details).

2. Explicit relativistic terms in $L^{i}$ results in additional periodic relativistic effects amounting to $\sim 1$ microarcsecond (see, Bizouard, et al., 1992). It is to note, however, that the analogous effect in the rotation of the Moon is $2-3$ order of magnitude larger and may amount to $\sim 1$ mas (see, Bois, Vockrouhlicky, 1995).

3. The key relation of Newtonian mechanics

$$
\left(M^{i j}\right)_{\text {Newton }}=\left(\frac{1}{3} \delta^{i j} C^{s s}-C^{i j}\right)_{\text {Newton }}
$$

relating the quadrupole moment of the gravitational field $M^{i j}$ and the tensor of inertia $C^{i j}$ can be shown to be violated in general relativity. This means that, for example, $J_{2}$, related to $M^{i j}$, cannot be easily related to the dynamical ellipticity $H$, which is derived from $C^{i j}$, the effect being of order of $\delta J_{2} / J_{2} \sim 10^{-9}$ (see, Klioner, 1997).

\section{References}

Bizouard C., Schastok, J., Soffel M.H. and Souchay J. (1992) Etude de la rotation de la Terre dans le cadre de la. relativité générale: premiere approche. In: Proc. Journées Systèmes de Référence Spatio-temporels 1992, ed. N Capitaine, Observatoire de Paris, pp. 76.

Bois, E. and Vockrouhlicky, D. (1995) Relativistic spin effects in the Earth-Moon system. Astron. Astrophys., 300, pp. 559.

Brumberg V.A. (1991) Essential Relativistic Celestial Mechanics. Adam Hilger, Bristol.

Brumberg V.A., Bretagnon P. and Guinot, B. (1996) Astronomical Units and Constants in the General Relativity Framework. Celest. Mech. Dyn. Astron., 64, pp. 231.

Damour T., Soffel M. and Xu Ch. (1991) General-Relativistic Celestial Mechanics. Phys. Rev. D., 43, pp. 3273

Damour T., Soffel M. and Xu Ch. (1992) General-Relativistic Celestial Mechanics. Phys. Rev. D., 45, pp. 1017

Damour T., Soffel M. and Xu Ch. (1993) General-Relativistic Celestial Mechanics. Phys. Rev. D., 47, pp. 3124.

Klioner, S.A. (1996) Angular Velocity of Rotation of Extended Bodies in General Relativity. In: Dynamics, ephemerides and astrometry in the solar system, eds. S. Ferraz-Mello, B. Morando and J.E. Arlot, Kluwer, Dordrecht, pp. 309

Klioner, S.A. (1997) On the problem of post-Newtonian Rotational Motion. In: Dynamics and Astrometry of Natural and Artificial Celestial Bodies, eds. I.M. Wytrzysczak, J.H. Lieske and R.A. Feldman, Kluwer, Dordrecht, pp. 383.

Soffel, M. (1994) The problem of rotational motion and rigid bodies in the post-Newtonian framework. Impublished notes. 